\title{
Determination of proliferative activity in nasal polyps
}

Sergio Hassid, Marie-Pierre Degaute, Sandra Dawance, Katja Rombaut, Nathalie Nagy, Georges Choufani, Christine Decaestecker, André Danguy, Isabelle Salmon, Robert Kiss

Service d'Oto-RhinoLaryngologie, Cliniques Universitaires de l'Hôpital Erasme, Belgium

$S$ Hassid

S Dawance

G Choufani

Service d'Anatomie Pathologique

M-P Degaute

K Rombaut

N Nagy

I Salmon

\section{Laboratoire}

d'Histologie, Faculté de Médecine,

Université Libre de

Bruxelles, Brussels,

Belgium

C Decaestecker

A Danguy

R Kiss

Correspondence to: Dr Kiss, Laboratoire d'Histologie, Faculté de Médecine, Université Libre de Bruxelles, 808 route de Lennik, 1070 Brussels,

Belgium.

Accepted for publication 2 September 1997

\begin{abstract}
Aims-To determine the level of proliferative activity in 39 nasal polyps with clear cut distinct clinical behaviour patterns.

Methods-The 39 nasal polyps included 11 polyps labelled as "single" and taken from the lateral nasal wall and the middle turbinate; 12 polyps labelled as "massive" and relating to diffuse polyposis involving the entire nasal cavity; six polyps labelled as "ASA" and relating to nasal polyps from patients with acetylsalicylic acid intolerance and asthma; and 10 polyps from cystic fibrosis related polyposis. Cell proliferation was determined by two independent methods: first, the computer assisted microscope analysis of isolated Feulgen stained nuclei for the measurement of the percentage of cells in the S phase of the cell cycle; and second, the immunohistochemical evaluation of a proliferation associated protein by means of the MIB 1 monoclonal antibody.

Results-The cystic fibrosis related polyposis exhibited the highest proliferative activity of all the clinically identified nasal polyp groups. Acute inflammatory nasal polyps exhibited a higher cell proliferation than chronic ones. The results also show that while the immunohistochemical determination of cell proliferation by means of the MIB 1 monoclonal antibody is a valuable tool in determining cell proliferation in nasal polyps, the cytometrical image analysis of Feulgen stained nuclei is not useful for this purpose.

Conclusion-Cell proliferation activity identifies cystic fibrosis as being distinct from the other nasal polyp groups.

(7 Clin Pathol 1997;50:923-928)
\end{abstract}

Keywords: nasal polyps; proliferation; immunohistochemistry; Feulgen; image cytometry

Nasal polyposis is a common disease that severely affects daily life in most sufferers. ${ }^{1}$ For some authors ${ }^{2}$ this disease constitutes a clinical problem rather than a histological one. Bernstein $e t a l^{3}$ state that in spite of recent advances in basic science, particularly immunology and molecular biology, the cause and pathogenesis of nasal and paranasal polyposis have not been clarified. These authors report ${ }^{3}$ that, in their opinion, although theories involving the dysfunction of the autonomic nervous system of the nose $\mathrm{e}^{4}$ as well as abnormalities in carbohydrate metabolism ${ }^{5}$ and vasomotor imbalance ${ }^{5}$ have been put forward, the two most plausible theories involve allergy ${ }^{6}$ and inflamation. ${ }^{7}$ In addition to all these data, it should also be emphasised that, as stated by Coste $e t$ al, ${ }^{1}$ respiratory epithelium (and indeed any lining epithelium) is subject to a continuous turnover that requires epithelial cell proliferation and differentiation for cell renewal and mucosal repair after various forms of aggression. ${ }^{8}$

Recently, we proposed a novel classification of nasal polyps. ${ }^{9}$ This classification relies on both morphological criteria relating to morphonuclear features from isolated Feulgen stained nuclei (that is, quantitatively describing the chromatin patterns) and glycohistochemical characteristics from histological slides treated with three lectins (peanut agglutinin (PNA isolated from Arachis hypogaea), wheat germ agglutinin (WGA isolated from Triticum vulgaris), and gorse seed agglutinin (UEA-I isolated from Ulex europaeus)) and one neoglycoconjugate carrying a $\mathrm{Gal}(\beta 1-3)$ GalNAc glycan residue, therefore related to the ThomsenFriedenreich antigen (TF antigen), which represents the acceptor sites of endogeneouslike PNAs. ${ }^{10}$ The data obtained from these studies showed that our morphological classification of nasal polyps fitted in with the classification of nasal polyps defined on the basis of clinical criteria. ${ }^{9}$

In our present study, we refer to the abovementioned nasal polyp classification ${ }^{9}$ and investigate whether any significant differences exist between the clinically related groups in terms of proliferation. Proliferative activity was determined by means of two independent methods. The first involved the use of computer assisted microscopy applied to Feulgen stained nuclei in order to quantify the proportion of cells present in the $S$ phase of the cell cycle (S phase fraction, SPF index). ${ }^{11}$ The second method was an immunohistochemical one which involved the use of the MIB 1 antibody that recognises cell proliferation associated human nuclear antigens (the $\mathrm{Ki}-67$ antigen) present in the late $G_{1}, S, G_{2}$, and $M$ phases of the cell cycle, ${ }^{12}{ }^{13}$ but absent in the $G_{0}$ and early $G_{1}$ phases. The MIB 1 monoclonal antibody reacts with recombinant parts of the $\mathrm{Ki}-67$ antigen (the 1002 base pair Ki-67 cDNA fragment). ${ }^{13}$

\section{Methods}

HISTOPATHOLOGICAL DIAGNOSIS AND CLINICAL DATA

Between 1993 and 1996 the medical records and samples of nasal polyps were obtained for 39 
patients. The diagnosis of polyposis was made with a rigid endoscope. This method enabled us to differentiate between single polyps (the single group; $n=11$ ) found typically between the lateral nasal wall and the middle turbinate, and diffuse polyposis (the massive group; $n=12$ ), which involves the entire nasal cavity. The ASA group $(n=6)$ included nasal polyps from patients with acetylsalicylic acid intolerance established by clinical history. Provocation tests were not performed. The cystic fibrosis related polyposis group ( $\mathrm{CF}$ group; $\mathrm{n}=10$ ) included patients with well documented pathologies and positive sweat tests.

The histological slides of all the cases were reviewed by the same pathologist on the basis of the criteria described by Hyams et al. ${ }^{14}$ The histological characteristics in terms of acute versus chronic inflammatory polyps were also reviewed in the light of the criteria described by these authors. ${ }^{14}$

\section{SPECIMEN PREPARATIONS}

The nasal polyp cases came from archive materials (formalin fixed, paraffin wax embedded tissues). One paraffin wax block was available for each case. Seven slides were cut from each block. The slides $1,3,4,5$, and 7 were $5 \mu \mathrm{m}$ thick. The slides 1 and 7 were haematoxylin and eosin stained for histological diagnosis. The slides 3 and 5 were immunostained by means of the anti-MIB 1 antibody (Immunotech SA (Marseille, France), code no. 0505, clone MIB 1 , dilution $1 / 50$ ). Slide 4 served as a negative control (the primary antibody was absent) for slides 3 and 5 . The procedure used was identical to the one detailed elsewhere. ${ }^{15}$ Slides 2 and 6 were $80 \mu \mathrm{m}$ thick and were sub-

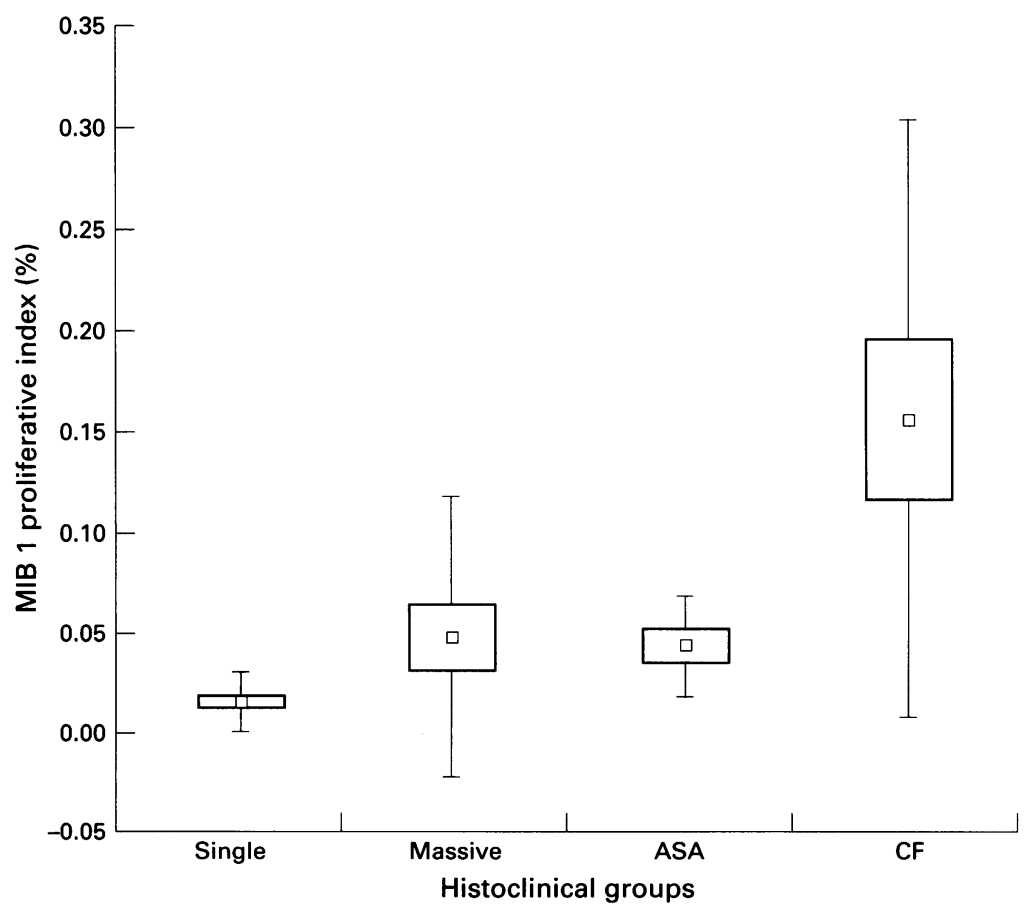

Figure 1 Development of the mean (small squares), standard error (large rectangles), and standard deviation (bars) values relating to the immunohistochemical MIB 1 labelling index in four nasal polyp groups. Single, polyps from the lateral nasal wall and the middle turbinate $(n=11)$. Massive, diffuse polyposis involving the entire nasal cavities $(n=12)$. $A S A$, polyps from patients with acetylsalicylic acid intolerance $(n=6)$. CF, polyps from patients with cystic fibrosis $(n=10)$. jected to a method that enables cell suspensions to be obtained (after pronase digestion). ${ }^{16}$ They were cytocentrifuged on to glass slides and submitted to the Feulgen reaction ${ }^{16}$ to determine the SPF index ${ }^{11}$ (see below).

\section{VARIABLE DETERMINATION}

$M I B 1$ index

For each of the 39 nasal polyps under study the percentage of positively labelled MIB 1 cells was recorded in 10 independent areas of the surface (lining) epithelium on each of the two slides available for any given case. Each area included between 800 and 1200 cells at a $\times 200$ magnification. The MIB 1 index represented the number of positively labelled MIB 1 cells divided by the total number of cells counted (positive and negative).

\section{SPF index}

The nuclear DNA content of each nasal polyp under study was assessed by means of a DNA histogram computed on 300 Feulgen stained cell nuclei. The computation was carried out by means of a SAMBA 2005 system (AlcatelTITN, Grenoble, France) on the basis of a procedure detailed elsewhere. ${ }^{11} 16$ The SAMBA system was equipped with a black and white CCD camera and a Leitz (Diaplan; Brussels, Belgium) microscope with a $\times 1000$ magnification (numerical aperture: 1.30).

The proliferation index is the percentage of cells engaged in the $S$ phase of a cell cycle. Thus, the proliferation index corresponds to the area under the curve between the $G_{1}$ and $G_{2}$ peaks in the DNA histogram. The proliferation index was calculated on each DNA histogram in accordance with the procedure that we have developed and which is detailed elsewhere. ${ }^{11}$

\section{DATA ANALYSIS}

The results are given in the figures as the mean with standard deviations and standard errors. The statistical comparisons of the data were performed by means of either one way variance analysis (the Fisher F test) when the value distribution was normal (checked by means of the $\chi^{2}$ test) and the variances equal (checked by the Bartlett test), or by the Mann-Whitney U test when the variances among groups analysed were not equal. All the statistical analyses were carried out using the Statistica/Dos software (Statsoft, Tulsa, Oklahoma, USA).

\section{Results}

RELATION BETWEEN HISTOCLINICAL TYPE AND PROLIFERATIVE ACTIVITY

Figure 1 shows the cell proliferation level as assessed by means of MIB 1 immunohistochemistry on the four nasal polyp groups under study.

The cell proliferation index in the single group was significantly lower than in the other three groups: $\mathrm{p}=0.02 v$ massive; $\mathrm{p}=0.01 v$ ASA; $p=0.0002 v$ CF. The CF group exhibited a significantly higher cell proliferation index than the remaining three groups: $\mathrm{p}=0.04 v$ massive; $\mathrm{p}=0.02 v$ ASA. The massive and ASA polyps exhibited a similar cell proliferation index $(p>0.05)$. 


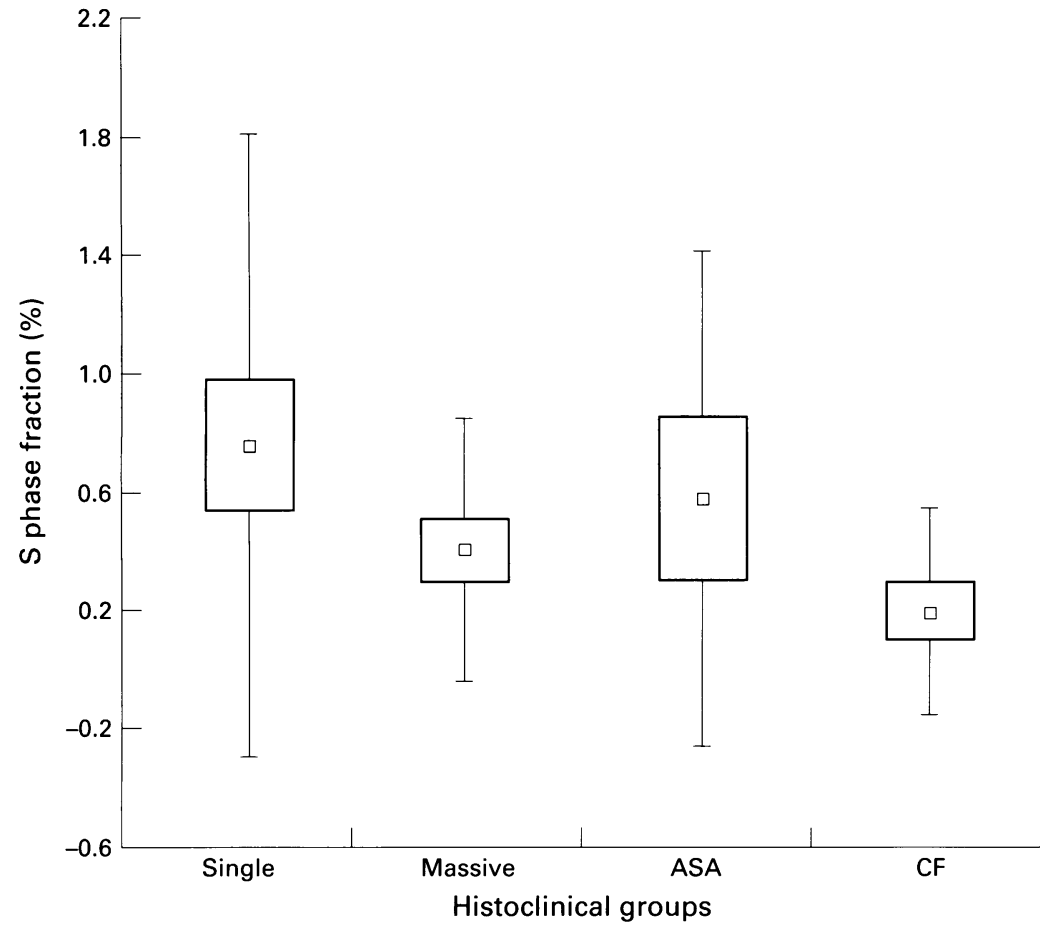

Figure 2 Development of the mean (small squares), standard error (large rectangles), and standard deviation (bars) values relating to the determination of the $S$ phase fraction by means of the cytometrical image analysis of Feulgen stained nuclei in the four nasal polyp groups described in fig 1 .
Figure 2 shows that no statistically significant $(p>0.05)$ differences were found between the various nasal polyp groups in terms of proliferative activity when this activity was determined by means of the SPF index.

An evident misfit is apparent between the data obtained when figs 1 and 2 are compared. This misfit might be explained, at least in part, as follows. Figure $3 \mathrm{~A}-\mathrm{C}$ illustrates the various patterns of MIB 1 immunostaining that can be obtained in nasal polyps. In fig $3 \mathrm{~A}$, only a few epithelial cells are labelled positively by the MIB 1 monoclonal antibody. In fig 3B and C, numerous epithelial cells are labelled, but the labelling pattern is distinct. In fig $3 \mathrm{~B}$, the labelling pattern is homogenous. Indeed, the density of the positive MIB 1 cells is homogenous in the lining epithelium. In contrast, in fig $3 \mathrm{C}$ the density of the positive MIB 1 cells is heterogenous, that is, there is a clonal growth pattern.

Figure 3D illustrates the type of biological material on which the SPF index was determined (the arrows indicate the cells that will be retained in the final analysis).

When the specimens shown in fig $3 \mathrm{~A}-\mathrm{C}$ are compared, it seems that the distribution of the proliferating cells in the lining epithelium is very heterogenous from one polyp to another. This means that the architecture of the lining epithelium must be retained if the proliferation
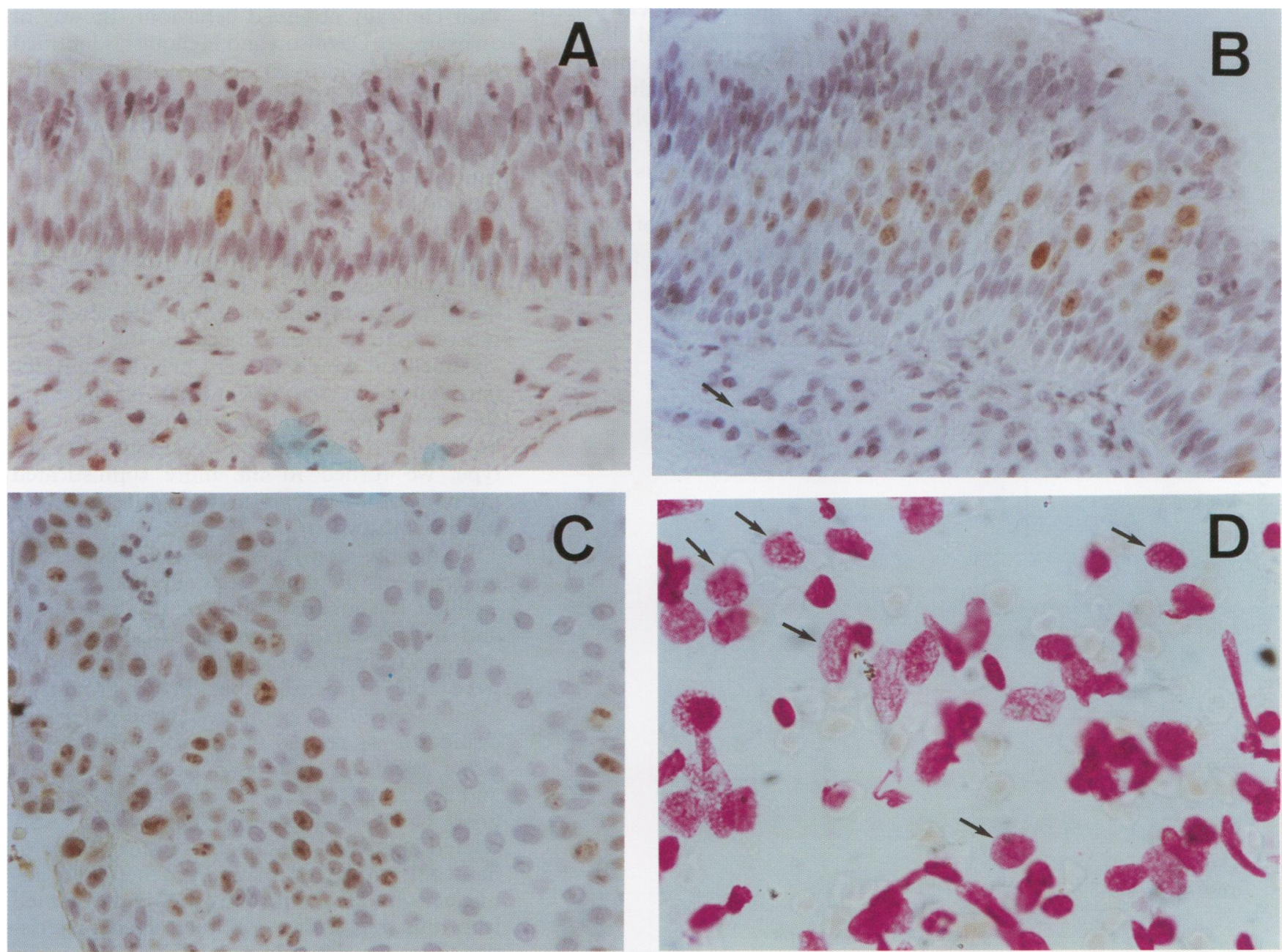

Figure 3 Illustration of cell proliferative activity as revealed by the MIB 1 monoclonal antibody in three nasal polyps. A nasal polyp whose surface epithelium is associated with a very low level of proliferation $(A)$. Two nasal polyps with a high level of proliferative activity in the surface epithelium showing a diffuse pattern of proliferation $(B)$ and a clonal pattern of proliferation $(C)$. A Feulgen stained cytospin to be submitted to computer assisted microscopy for determination of the $S$ phase fraction (D). The arrows indicate those nuclei that will be retained in the final analysis. 

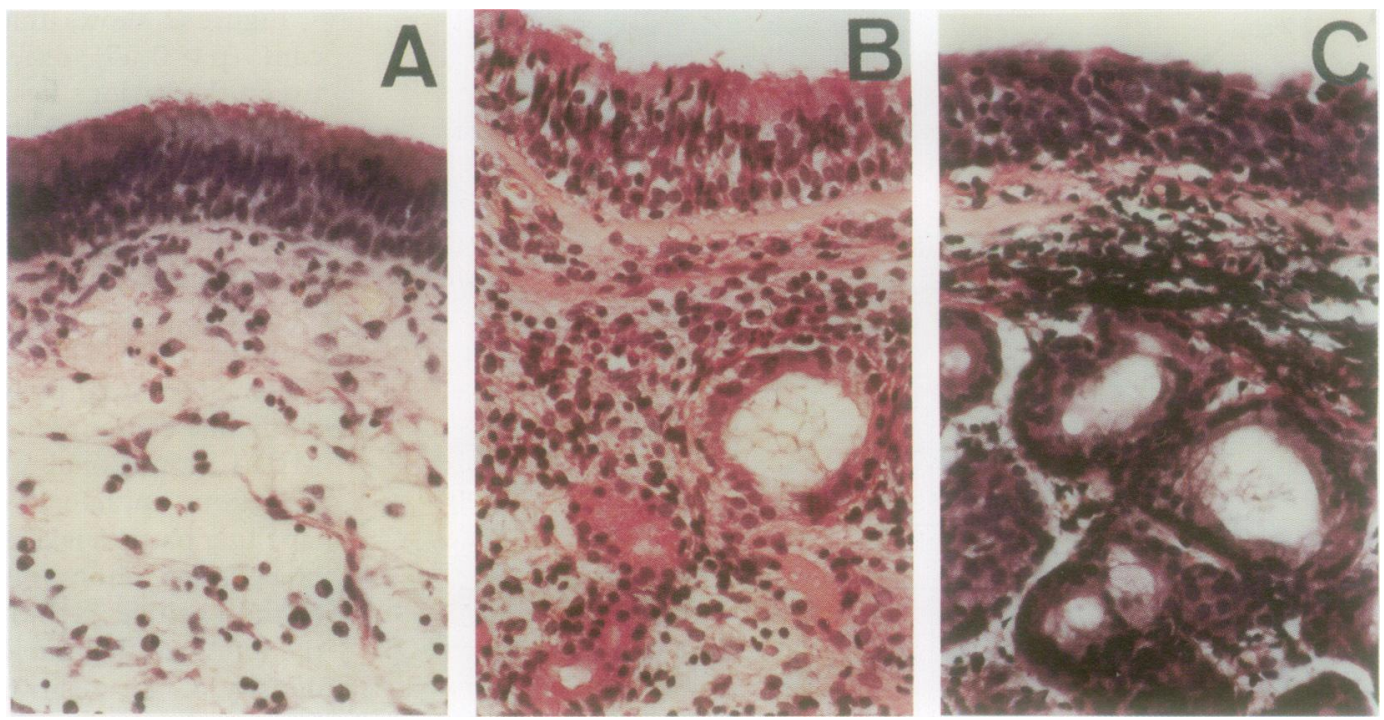

Figure 4 Histological patterns (haematoxylin and eosin staining; original magnification, $\times 200$ ) of an acute inflammatory nasal polyp, characterised by the absence of glands in the stroma $(A)$, and two chronic inflammatory nasal polyps, characterised by the presence of either a few (B) or many $(C)$ seromucinous glands in the stroma.

rate is to be determined correctly. Figure 3D shows clearly that this architecture no longer existed in the biological sample submitted for analysis to the computer assisted microscope.

\section{RELATION BETWEEN INFLAMMATORY STATE AND PROLIFERATIVE ACTIVITY}

Figure 4A illustrates the histological pattern (on haematoxylin and eosin stained slides) of an acute inflammation nasal polyp, characterised by the absence of glands in the stroma. Two chronic inflammation nasal polyps, characterised by the presence of either a few (fig $4 \mathrm{~B}$ ) or many (fig 4C) seromucinous glands in the stroma are also shown.

Figure 5 shows that the pattern of proliferative activity is significantly different $(p=0.02)$

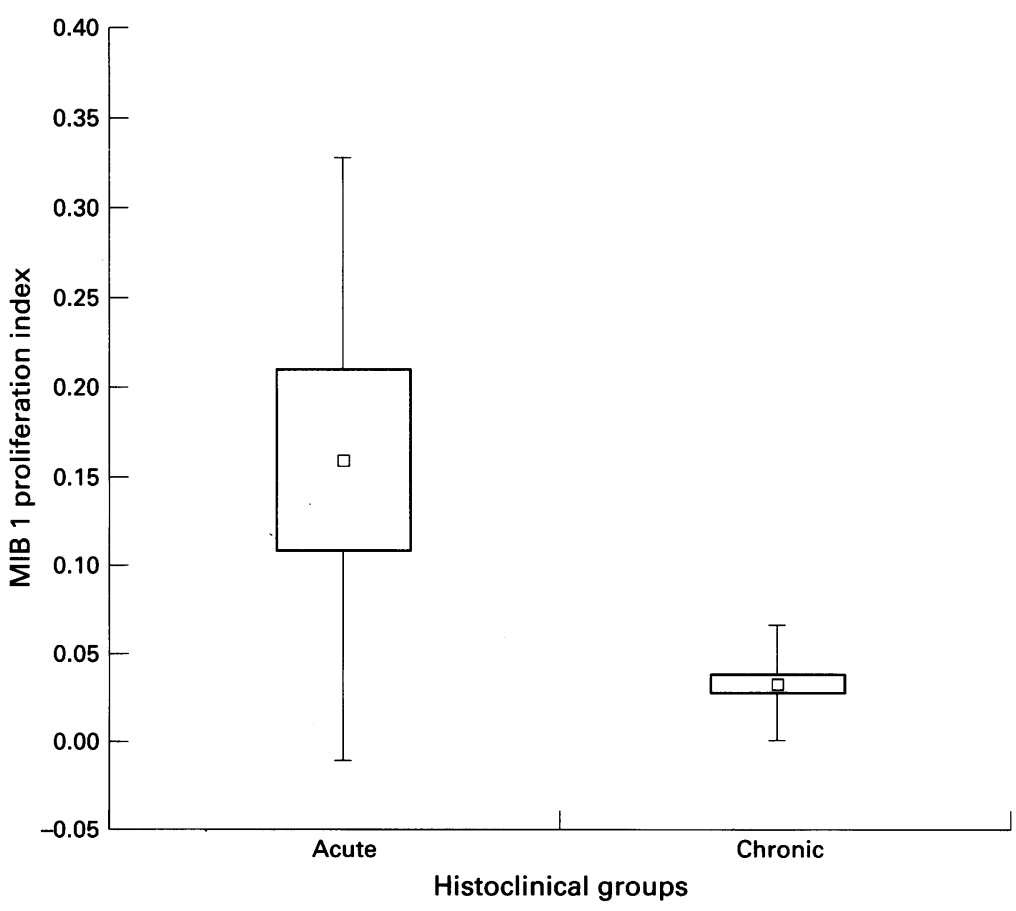

Figure 5 Development of the mean (small squares), standard error (large rectangles), and standard deviation (bars) values relating to the MIB 1 immunohistochemical labelling index in acute versus chronic inflammatory nasal polyps as described in fig 4. in these two groups of acute versus chronic inflammatory nasal polyps.

\section{Discussion}

The morphological appearance of the nasal polyps on the $5 \mu \mathrm{m}$ thick haematoxylin and eosin stained histological slides did not reflect their clinically observed behaviour. By way of an example, Davidson and Hellquist ${ }^{2}$ identified four morphological types of nasal polyp: common; chronic inflammatory; a type showing hyperplasia or seromucinous glands; and a type with atypical stroma-a nasal polyp type that can be mistaken for a neoplasm. ${ }^{2}$ These four morphologically identified nasal polyp types did not fit in with our study of the four nasal polyp groups selected on the basis of clinical criteria. ${ }^{9}$ Therefore, we attempted to establish a nasal polyp classification on the basis of morphological criteria because this type of classification would be very helpful clinically, particularly in the monitoring of patients. ${ }^{9}$ Because conventional morphology appears to be insufficient to set up a classification of this type, we turned to the more sophisticated methods of image cytometry and lectin glycohistochemistry.

Using the computer assisted microscope analysis of Feulgen stained nuclei (that enabled the morphometry of the cell nuclei and the chromatin patterns to be described quantitatively by means of 26 variables), we were able to identify four major groups of nasal polyps: diffuse allergy related polyposis, cystic fibrosis related polyposis, single polyps either associated or unassociated with allergy, and a fourth group that included diffuse polyposis not associated with allergy and ASA related polyposis. ${ }^{17}$ Thus, these four groups of nasal polyps differed markedly in their morphonuclear characteristics, but the data obtained from this first study took nuclear characteristics into consideration only. Therefore, we completed our investigation by a second study that characterised the cytoplasmic and membrane related properties demonstrated by 
means of lectin and néoglycoprotein histochemistry. ${ }^{10}$ We observed that the group of single polyps exhibited distinct glycohistochemical characteristics when compared with both diffuse and cystic fibrosis related polyposis groups. ${ }^{10}$ It was by combining these two methodological approaches (nuclear Feulgen staining and cytoplasmic and membrane glycohistochemical staining) that we succeeded in setting up the abovementioned morphology based classification for nasal polyps. $^{9}$

The aim of the present study was, therefore, to investigate whether the four groups of clinically identified nasal polyps exhibited distinct patterns of proliferative activity.

At the present moment, it remains unclear how a polyp develops from normal nasal mucosa. As mentioned, of all the theories put forward, the predominate one concerns major defects (probably induced by inflammatory processes) that occur in the bioelectric integrity of the $\mathrm{Na}^{+}$and $\mathrm{Cl}^{-}$channels on the luminal surface of the epithelial respiratory cells. ${ }^{3}$ Bernstein $e t a l^{3}$ argue that a change in $\mathrm{Na}^{+}$ absorption may result in an increased movement of water into a cell and the interstitial fluid with the ultimate result that all these disturbances lead to an oedema that may, in turn, lead to the growth and enlargement (by swelling) of the surface epithelium into a nasal polyp. However, the theory that increased cell proliferation occurs in nasal polyps in comparison with normal mucosa cannot be ignored. Indeed, as stated by Coste et al, ${ }^{1}$ nasal polyps are not only characterised by the bulging of the oedematous process made up of a loose oedematous mucosal connective tissue, but also by the covering of this connective tissue by the respiratory epithelium that has an increased surface area compared with that of normal nasal mucosa.

Coste et $a l^{1}$ made use of two independent methods, flow cytometry and proliferation cell nuclear antigen (PCNA) immunohistochemistry, to determine the proliferative activity in a series of patients. For each patient, they compared the epithelial cell proliferation in nasal polyps and inferior turbinate nasal mucosa in which polyps never occur and reported that the percentage of cells in the $S$ phase was significantly higher $(p<0.05)$ in cell populations obtained from nasal polyps than cells from normal mucosa. ${ }^{1}$ However, they observed some misfits between the flow cytometry and immunohistochemistry related results. It should be remembered that although the flow cytometry method allows the automatic quantification of the nuclear DNA content on the basis of cell cycle analysis of a large number of individual cells (several thousand) in a few minutes, it requires the disintegration of the tissue to be analysed. This means that the tissue architecture is no longer respected and the so called epithelial compartment that, theoretically, is the only one analysed by flow cytometry, may be contaminated by many other cell types including inflammatory and connective tissue cells. In our present study, we made use of two methods similar to those used by Coste et al : image cytometry of Feulgen stained nuclei, which is similar to flow cytometry and MIB 1 antigen immunohistochemistry, which is also an immunohistochemical method like PCNA. Our data show clearly that the disintegration of the tissue required for image cytometry analysis did not enable us to demonstrate any specific proliferation pattern in the various nasal polyp groups while the MIB 1 immunohistochemical method did enable this distinction to be made. This feature relies directly on the particular growth pattern of the surface epithelium in nasal polyps, as shown in fig 3 .

Thus, our results show that among all the clinically identified nasal polyp groups, it was the cystic fibrosis related ones that exhibited the highest proliferative activity. Therefore, this biological feature identifies cystic fibrosis as being clearly distinct from the other nasal polyp groups. In a recent editorial, Batsakis and El-Naggar ${ }^{18}$ wrote: "can sinonasal polyps from patients with cystic fibrosis be distinguished from atopic nasal polyps? Probably not”. It seems that the biological markers from the morphological field that we have detailed elsewhere, ${ }^{910}$ and those relating to the cell proliferative activity detailed here, could help in distinguishing between these two groups of nasal polyps. The present study also shows that acute inflammatory nasal polyps exhibit a higher level of cell proliferation than chronic ones.

This work was supported by grants awarded by the Fonds de la Recherche Scientifique Médicale (FRSM, Belgium)

1 Coste A, Rateau JG, Roudot-Thoraval F, Chapelin C, Gilain $\mathrm{L}$, Poron F, et al. Increased epithelial cell proliferation in nasal polyps. Arch Otolaryngol Head Neck Surg 1996;122:432-6.

2 Davidson A, Hellquist HB. The so-called "allergic" nasa polyp. Otorhinolaryngology 1993;55:30-5.

3 Bernstein J, Gorfien J, Noble B. Role of allergy in nasa polyposis: a review. Otolaryngol Head Neck Surg 1995;113: 724-32.

4 Cauna N, Hinderer KH, Manzetti CW, Swanson EW. Fine structure of nasal polyps. Ann Otol Rhinol Laryngol 1972;81:41-58.

5 Slavin RG. Nasal polyps and sinusitis. In: Middleton E Jr, Reed CE, Ellis CE, Atkinson NF Jr, Yunginger JW, eds. Reed CE, Ellis CE, Atkinson NF Jr, Yunginger JW, eds.
Allergy: principles and practice, Vol 3, 3rd edn. St Louis: CV Allergy: principles and practic

6 Holopainen E, Makinen J, Paavolainen M, Palva T, Saldo DR. Nasal polyposis: relationship to allergy and acetyl salicylic acid intolerance. Acta Otolaryngology 1979;87: 330-4

7 Small P, Frenkiel S, Black M. Multifactorial etiology of nasal polyps. Ann Allergy 1981;46:317-20.

8 Breeze RG, Wheeldon EB. The cells of the pulmonary airways. Annu Rev Respir Dis 1977;116:705-777.

9 Hassid S, Decaestecker C, Hermans C, Salmon I, Pasteels $\mathrm{JL}$, Danguy A, et al. Lectin glycohistochemical and Feulgen cytometrical characteristics analyzed using an artificial intelligence-related algorithm to propose a new histocliniintelligence-related algorithm to propose a new histoclinical classification

Laryngol. [In press.]
Hassid S, Salmon I, Brugmans M, Dawance S, Kiss R Gabius HJ, et al. Histochemical study of epithelia of nasa polyps by biotinylated lectins and neoglycoproteins. A comparison with the normal respiratory epithelium. Eur Morphol 1997;35:77-85.

11 Salmon I, Kiss R. Relationship between proliferative activity and ploidy level in a series of 530 human brain tumors including astrocytomas, meningiomas, schwannomas and metastases. Hum Pathol 1993;24:329-35.

12 Gerdes J, Schwab U, Lemke $\mathrm{H}$, Stein $\mathrm{H}$. Production of mouse monoclonal antibody reactive with a human nuclear mouse nonoclinal anth cell proliferation. Int $\gamma$ Cancer

13 Cattoretti G, Becker MH, Key G, Duchrow M, Schulter C, Galle J, et al. Monoclonal antibodies against recombinant parts of the Ki-67 antigen (MIB 1 and MIB 3) detect proliferating cells in microwave-processed formalin-fixed paraffin sections. $\mathcal{f}$ Pathol 1992;168:357-63. 
14 Hyams VJ, Batsakis JG, Michaels L. Nasal and paranasal polyposis. In: Hyams VJ, Batsakis JG, Michaels L, eds. Tumors of the upper respiratory tract and ear, 2 nd edn. Atlas of Tumor Pathology. Washington DC: Armed Forces Institute of Pathology, 1988;25:11-33.

15 Kiss R, DeWitte O, Decaestecker C, Camby I, Gordower L, Delbecque $\mathrm{K}$, et al. The combined determination of prolifDative activity and cell densi 作 to long-surviving adult patients with supratentorial high-

$321-31$
16 Kiss R, Salmon I, Camby I, Gras S, Pasteels JL. Characterization of factors in routine laboratory protocols which significantly influence the Feulgen reaction. $f$ Histochem Cytochem 1993;41:935-45.

17 Hassid S, Choufani G, El-Kattabi O, Dawance S, Decaestecker $C$, Brugmans $M$, et al. Image cytometry characterization of ploidy level, proliferative activity and chromatin pattern in 50 nasal polyps. Int $\mathcal{F}$ Oncol 1996;9:137-43.

18 Batsakis JG, El-Naggar AK. Pathology consultation. Cystic fibrosis and the sinonasal tract. Ann Otol Rhinol Laryngol $1996 ; 105 \cdot 329-30$ 